\title{
COHERENT PHONON SPECTROSCOPY OF SEMICONDUCTOR-METAL INTERFACES
}

\author{
Y.-M. Chang \\ Center for Condensed Matter Sciences, National Taiwan University \\ No. 1, Sec. 4, Roosevelt Rd., Taipei 10673, Taiwan, R.O.C. \\ Tel: 886-2-33665277 Fax: 886-2-23655404. E-mail: ymchang@ccms.ntu.edu.tw
}

\begin{abstract}
Coherent LO and LA phonons of GaP-Au Schottky interfaces are probed with TRSHG technique. The driving mechanisms for these phonon modes are proposed. Bias-dependent carrier and phonon interaction can now be investigated in femtosecond timescale.
\end{abstract}

The development of advanced femtosecond laser has made it possible to drive coherent phonons and detect their oscillations in various condensed matters with optical pump-probe technique [1]. The phonon frequency and dephasing time can be directly determined from the temporal domain data. In this paper, we demonstrate the coherent phonon spectroscopy of GaP using a simple semiconductor-metal structure and time-resolved second-harmonic generation (TRSHG) technique [2]. The studied sample structure is: $10 \mathrm{~nm}$ Au Schottky layer, $30 \mu \mathrm{m}$ N-type GaP layer $\left(\mathrm{n}_{\mathrm{d}}<10^{16} / \mathrm{cm}^{3}\right)$, N-type GaP $(100)\left(\mathrm{n}_{\mathrm{d}} \sim 5 \times 10^{17} / \mathrm{cm}^{3}\right)$, and metal electrode.

Fig. 1 shows a typical TRSHG measurement with higher time resolution $(6.6 \mathrm{fs})$. The oscillatory component can be extracted from the slowly varying background and analyzed with Fourier transform. Its Fourier spectrum is shown in the inset and reveals a peak at $12.2 \pm 0.1 \mathrm{THz}$, which agrees with the LO phonon frequency of GaP. The oscillatory component is further curve-fitted to determine its phonon amplitude and dephasing time [2]. On the other hand, TRSHG measurement with longer scan range but lower time resolution (264 fs) is plotted in Fig. 2, where a long periodical oscillation can be clearly identified. The frequency and decay time are determined to be $122 \pm 8 \mathrm{GHz}$ and $16.0 \pm 0.5 \mathrm{ps}$. This low frequency mode is assigned to coherent LA wave packet propagating into GaP bulk. We furthermore perform the pump power and polarization dependent, and bias voltage dependent TRSHG measurements to determine the possible driving mechanisms and dephasing processes of these phonon modes.

Our experimental results indicate that impulsive stimulated Raman scattering and transient depletion field screening can both play roles in the generation of coherent LO phonon. At reverse or zero bias voltage, the LO phonon dephasing time is about $9 \sim 11 \mathrm{ps.} \mathrm{The} \mathrm{dephasing} \mathrm{process} \mathrm{is} \mathrm{mainly} \mathrm{due} \mathrm{to} \mathrm{anharmonic} \mathrm{decay} \mathrm{into} \mathrm{two}$ zone-edge half-energy LA acoustic phonons: $\mathrm{LO}\left(\omega_{\mathrm{LO}}, 0\right) \rightarrow \mathrm{LA}\left(\omega_{\mathrm{L} \sigma} / 2, q\right)+\mathrm{LA}\left(\omega_{\mathrm{LO}} / 2,-q\right)$. However, at forward bias, the LO phonon dephasing time decreased rapidly. This phenomenon is mainly due to severe carrier-phonon momentum scattering in the depletion region whenever a large DC current ( 0.15Amp) flows cross the metal-semiconductor interface. At the same time, coherent LA phonon is impulsively launched via transient thermal strain induced in the metal-semiconductor interface $[3,4]$ and then propagates into GaP bulk. The observed oscillatory period of LA phonon is determined by the formula [4]: $\tau=\lambda\left(2 v_{s} n\right)$, where $\lambda$ the probed wavelength, $v_{s}$ the LA sound velocity, and $n$ the refractive index of GaP. The obtained $v_{s}$ value, $\sim 5.8 \times 10^{5}$ $\mathrm{cm} / \mathrm{sec}$, shows excellent agreement with that obtained in typical Brillouin scattering measurement [5].

The author acknowledges the support of National Science Council, Ministry of Education and Academia Sinica of Taiwan, R.O.C.

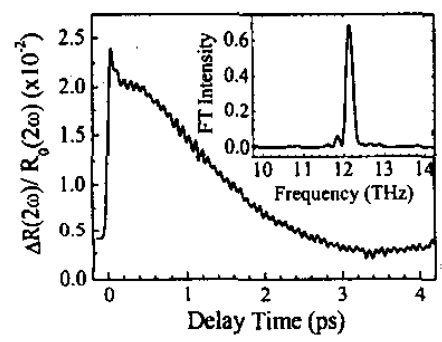

Fig. 1

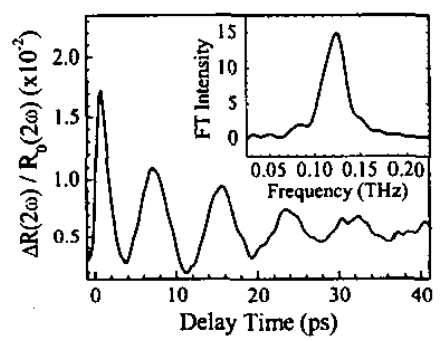

Fig. 2

[1] T. Dekorsy, G C. Cho, and H. Hurz, in Light Scattering in Solids VIII (Springer, Berlin, 2000), and references therein.

[2] Y.-M. Chang, Appl. Phys. Lett. 80, 2487 (2002).

[3] J. S. Yahng, Y. D. Jho, K. J. Yee, E. Oh, J. C. Woo, and D. S. Kim, Appl. Phys. Lett. 80, 4723 (2002).

[4] C. J. Stanton, G D. Sanders, R. L. Liu, C. S. Kim, J. S. Yahng, E. Oh, D. S. Kim, and C.-K. Sun, in Ultrafast Phenomena in Semiconductors VI, Proc. SPIE Vol. 4643, edited by K. T. F. Tsen, J. J. Song, H. Jiang, (SPIE, Washington, 2002).

[5] M. Yamada, K. Wasa, and C. Hamaguchi, Jpn. J. Appl. Phys. 15, 1107 (1976).

\footnotetext{
0-7803-7766-4/03/\$17.00@2003 IEEE
} 